\title{
Multi-Channel MAC Protocol Using Statistical Channel Utilization for Cognitive Networks
}

\author{
Gao Xiang, Wen-Min Zhu and Hyung-Kun Park, Member, KIMICS
}

\begin{abstract}
Opportunistic spectrum access (OSA) allows unlicensed users to share licensed spectrum in space and time with no or little interference to primary users, with bring new research challenges in MAC design. We propose a cognitive MAC protocol using statistical channel information and selecting appropriate idle channel for transmission. The protocol based on the CSMA/CA, exploits statistics of spectrum usage for decision making on channel access. Idle channel availability, spectrum hole sufficiency and available channel condition will be included in algorithm statistical information. The model include the control channel and data channel, the transmitter negotiates with receiver on transmission parameters through control channel, statistical decision results (successful rate of transmission) from exchanged transmission parameters of control channel should pass the threshold and decide the data transmission with spectrum hole on data channel. The proposed protocol's simulation will show that proposed protocol does improve the throughput performance via traditional opportunistic spectrum access MAC protocol.
\end{abstract}

Index Terms - MAC protocol, spectrum hole prediction, utilization function, multi-channel data transmission.

\section{INTRODUCTION}

THE spectrum is allocated to licensed users with a static manner, while the spectrum demand nowadays for GSM, WLAN, WIMAX etc is increasing. The traditional allocation policy faces spectrum scarcity in particular spectrum bands. But under the observation of the Federal Communication Commission (FCC) [1], it was shown that over $70 \%$ of the allocated spectrum is not used at any given time even in a crowded area where the spectral usage is intensive. So the concept of cognitive radio has been proposed to make use of the unused spectrum at a given time and place. In cognitive radio networks, unlicensed (secondary) users can make use of licensed frequency bands without violating the licensed (primary) users. Also in cognitive radio wireless ad-hoc networks, secondary users do not have pre-assigned

Manuscript received May 22, 2010; revised May 26, 2010; accepted June 3, 2010.

The authors are with the Department of information \& Communication, Korea University of Technology and Education, Cheonan, 330-708, Korea (Email: flynn@kut.ac.kr) frequency bands but they opportunistically search, find and operate in an available and without constraining the primary users [2].

The appropriate cognitive radio MAC protocol need be designed according to the characteristic of cognitive radio ad-hoc networks. 3 important issues need be considered. Firstly, according to the sensing and channel statistical information, find the appropriate idle channels for second user. Secondly, it has to coexist with primary users. Thirdly, second user data transmission need avoid interference with primary, or reduce the interference below an acceptable level.

Some proposed papers have given us some ways to design the cognitive MAC protocol. But they have less focus on the channel condition information statistical [3] -[4]. Some one focus on the channel statistical infor mation, but the statistical is more complicated and $\mathrm{t}$ he statistical information is difficult to collect in act ual network [5]. We proposed a new cognitive radio MAC protocol for finding appropriate idle (white) channel, and using it for second user data transmission. The channel selecting algorithm will base on the series channel statistical information exchanged in control channel. And we will make sure that the interference that is induced by second user transmission below an acceptable level.

The remainder of the paper is organized as follows. Section 2 presents our proposed protocol, section 3 and section 4 provides system performance and simulation results, and in section 5, we will give the conclusion of our paper.

\section{CHANNEL STATISTICAL ALLOCATION PROTOCOL}

One of the most challenging tasks in developing opportunistic spectrum access (OSA) networks is the design of cognitive medium access control (MAC) protocols. The typical issues focus on how to find the white spectrum space, exchange and negotiate the channel statistical information, make idle channel allocation decision and avoid the interference with primary users in data transmission. According to it, our proposed protocol will be introduced by follows section: environment sensing, RTS/CTS exchange over control channel, and DATA/ACK transmission over data channel. 


\section{A. Overview of protocol}

The proposed protocol based on the CSMA/CA protocol, and the two kinds of channel (control channel and data channel) are included in it. For avoiding collision, control channel and data channel are added the collision avoidance windows before packet (control packet and data packet) transmission. Through exchange the channel statistical information with RTS and CTS in control channel, calculating and making decision of the idle channel allocation. Usable data packets are transmitted in negotiated and allocated idle channel, and end it as receiving the ACK packet in transmitter.

\section{B. Environment sensing}

Environment sensing is for finding the statistical information of each channel before true data transmission. In our protocol, it is not necessary to sense and learn all of the channels in spectrum. Each node is allocated a sensing range, which based on each node device's MAC address to spread the central channels of devices over the whole spectrum. It achieves spectrum load balancing nicely.

\section{RTS/CTS exchange over control channel}

When the node $\mathrm{A}$ has packets that the length is $L$ intended to node B. Node A listens to the control channel and waits until it becomes idle. Then, it waits for the channel to remain idle for DIFS duration before it begins the countdown of the contention window. If the channel is still idle after the contention window. A sends the RTS that includes the length of packet $L$ in the control channel.

When the node B receives the RTS packet, it begins to sense the data channels and find spectrum hole. For getting the spectrum hole time duration, we need use utilization in formation of primary user. Utilization of primary user can be got from environment sensing and learning. If we use the spectrum hole for transmission, the collision probability with the primary user must be below desired threshold $C_{t h}$.

The predicted time duration of spectrum hole is

$$
1-(1-\text { utilizep }(i))^{\text {timep }(i)} \leq C_{\text {th }}
$$

Where, utilizep $(i)$ is the utilization of legacy user in the unit time. The timep $(i)$ is the predicted idle time according to the collision with legacy user.

The receiver calculates the predicted data length that the spectrum hole can transmit during the idle duration.

$$
\text { length }(i)=\operatorname{time}(i) * \operatorname{rate}(i), i=0,1, \ldots, m
$$

Where, timep $(i)$ is the predicted time duration of spectrum holes of each available channel. The $m$ is the number of available channels.

After calculating, we can get a sequence where from $\max$ to min. the receiver stop choosing the channel until

$$
\sum_{0}^{K} \text { length }(i)>L, K<m
$$

Where, $K$ is the number of channels that is used for data transmission.

After the receiver makes the decision, B sends CTS to A with selected channels information. Then the A receivers the CTS, and A switches from control channel to the selected data channel and transmit the data immediately.

\section{DATA/ACK transmission over data channels}

Before the data channel transmission, a collision avoidance window for the coming transmission is designed. This back-off window is designed to reduce the probability of collision resulted by two transmission pairs that happen to select the same or overlapped data channels for transmission. After the collision avoidance window, if the data channel is also idle, the data will be transmitted, the process will be end as the sender received the ACK from the receiver.

If a collision happens on some selected channels, the data transmission will be stopped. After all selected channel's idle time duration expire, if all messages which length is $\mathrm{L}$ has been transmitted, the whole transmission is over. Otherwise, the algorithm go back to calculate the predicted idle time duration of spectrum hole, and continue to select other channel and renegotiate with destination.

\section{SYSTEM PERFORMANCE}

In this paper, we use collision probability and system throughput to analysis performance of system. If there is an appearance of primary user when the unlicensed user transmitting the data, thus causing the collision. We use passion distribution to analysis the collision probability. Passion distribution is a discrete probability that express the probability of a number of events occurring in a fixed period of time, if these events occur with known average rate

$$
f(k ; \lambda)=\frac{\lambda^{k} e^{-\lambda}}{k !}
$$

Where, $e$ is the base of the natural logarithm, usually $e=2.718$, the $K$ is the number of occurrences, and $\lambda$ is the average rate in the unit time.

The collision probability can be calculated by

$$
p_{c i}=\prod_{i=0}^{h}\left(1-f\left(0 ; \lambda_{i}\right)\right)
$$

Where, $h$ is the number of selected channel that has the random traffic, and $\lambda_{i}$ is the utilization of the legacy user in the unit time.

So, the system throughput can be described as 


$$
\text { syth }=\frac{\sum_{i=0}^{g}\left(t_{i} *\left(1-p_{c i}\right)\right)}{\tau}
$$

Where, $g$ is the number of channel that selected for data transmission, $\tau$ is the duration of whole data transmission, and the $t_{i}$ is the duration of data transmission of each selected channel.

\section{SIMULATION}

In simulation, we set one primary user and one secondary user in the network. For sampling time, $0.01 \mathrm{~s}$ was selected. The number of the channels are the 10 , and the average idle length of channel and average busy length of channel are the $[0.04,0.07]$ and $[0.07$, $0.1]$.

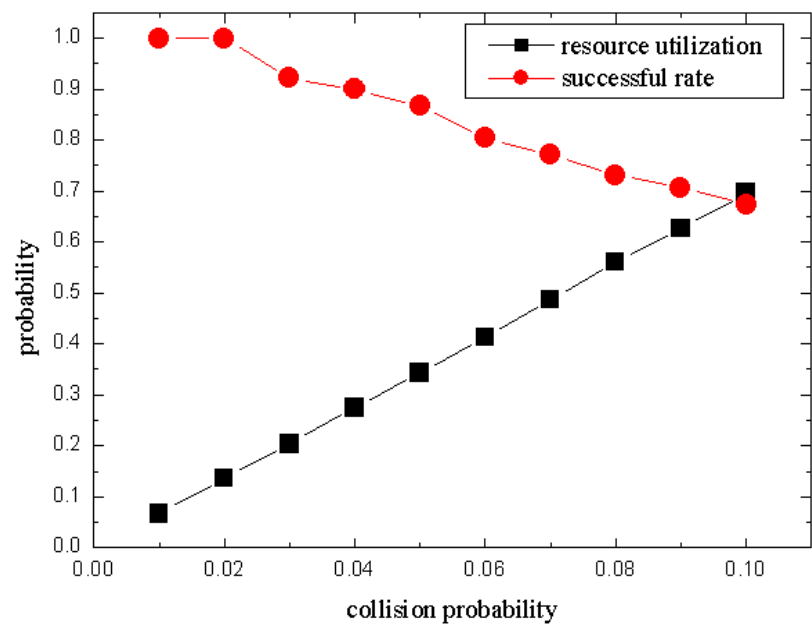

Fig. 1. The resource utilize and success rate vs. the number of the channel.

According to the collision probability, the resource utilization and successful rate are shown in figure 1. The resource utilization means that the ratio of sum of predicted idle time with sum of real idle time. From figure 1 , the successful rate decrease and the resource utilization increase when the collision probability threshold increases. Because when we select the larger threshold for collision threshold, the much more channel will be selected as idle channel, the resource utilization will increase, and collision probability also will increase, so the successful rate will decrease.

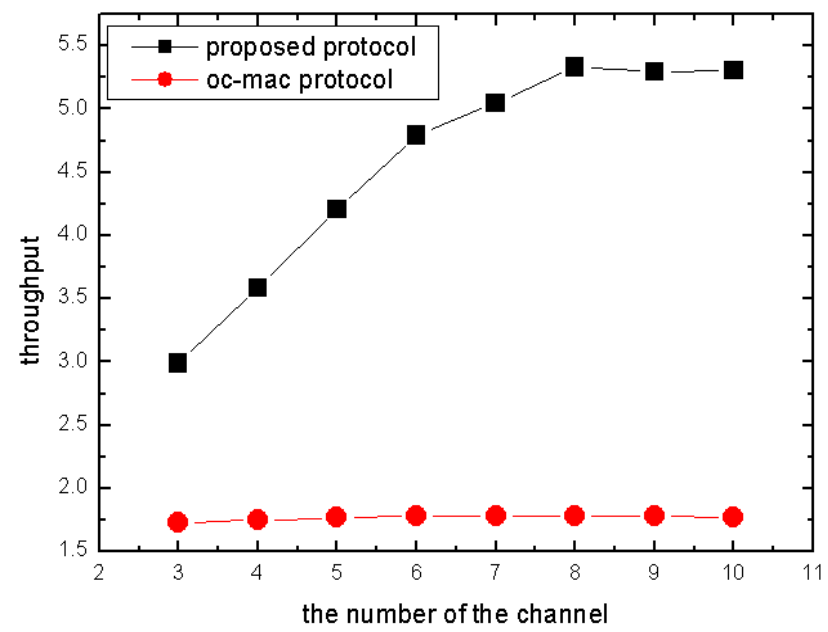

Fig. 2. The throughput in proposed algorithm and ocmac.

The figure 2 show us the throughput of proposed protocol vs. oc-mac protocol, according to the different number of channels. When the number of channel is more than 5 in the network, the throughput of proposed protocol is much larger than the oc-mac protocol, because the multi-channel was selected in proposed protocol.

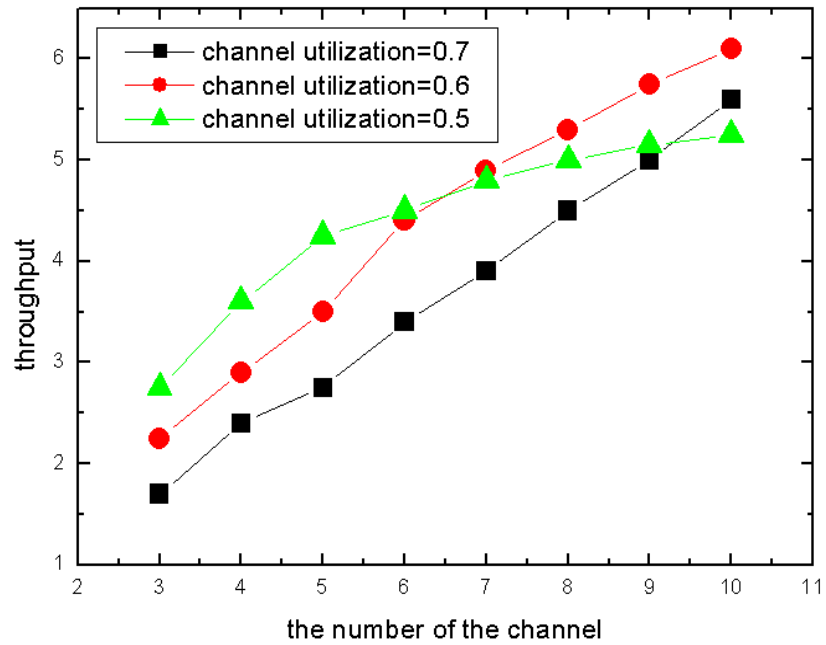

Fig. 3. According to channel utilization, the throughput in proposed algorithm and oc-mac.

In figure 3, we can see that as the number of channels increases, the throughput increases. This is because our proposed MAC protocol is multi-channel protocol, so the selected number of channels for transmitting increases as the number of channels increases. We also can see that when the number of channels is less, as the channel utilization increases, the throughput decreases. That is because the channel opportunities are not enough for secondary users when the number of channels is less and channel utilization is high. When the number of channels is large, the channel utilization increases, the throughput 
increases. This is because if the channel utilization is high, the predicted idle time is short. So the number of selected channels is large.

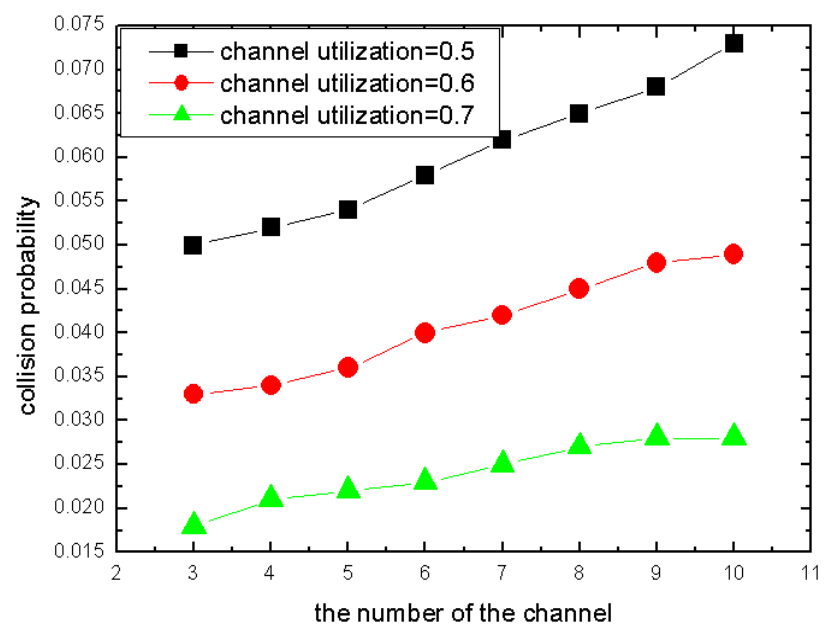

Fig. 4. According to channel utilization, collision probability in proposed algorithm and oc-mac.

In this simulation, we study the collision probability. we define that the collision probability is the probability that he predicted idle time smaller than the real idle time. In figure 4 , we can see that the collision probability will increase when the number of channels increases. This is because the channel opportunities increase as the number of channels increases. We can see that as the channel utilization increases, collision probability decreases. That is because if the channel utilization is large, the predicted idle time is small.

\section{CONCLUSIONS}

In this paper, we proposed a new MAC protocol in cognitive radio wireless ad-hoc network, by using channel statistical allocation. We show the protocol model and algorithm. By collecting the channel statistical information and calculating, the appropriate channel can be selected for second user transmission. Through the simulation results, we can get the better successful rate and throughput than the traditional MAC protocol.

\section{REFERENCES}

[1] FCC Spectrum Policy Task Force, "Report of the spectrum efficiency working group," Federal Communication, Technical Report 02-155, November 2002.

[2] S. Haykin, "Cognitive radio: brain-empowered wireless communications," IEEE JSAC, vol. 23, no. 2, pp. 201-220, February 2005.

[3] H, Su and X, Zhang, "Cross-layer based opportunistic MAC protocols for QoS provisionings over cognitive radio wireless networks," IEEE JSAC, Vol. 26, No. 1, pp. 118-129, January 2008 .
[4] Alex Chia-Chun Hsu, David S. L. Wei, and C.-C. Jay Kuo, "A cognitive MAC protocol using statistical channel allocation for wireless Ad-hoc networks," Proc. IEEE WCNC 2007, pp. 105-110, March 2007.

[5] Shao-Yi Hung and Yu-Chun Cheng, Eric Hsiao-Kuang Wu, and Gen-Huey Chen, "An opportunistic cognitive MAC protocol for coexistence with WLAN," Proc. IEEE ICC 2008, pp. 4059-4063, May 2008.

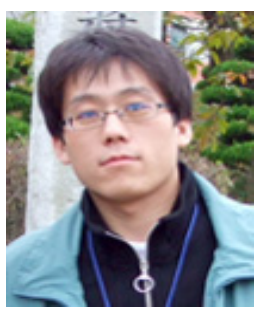

\section{Gao Xiang}

He received the B.S. degrees in the school of information engineering from Shandong University at Weihai in 2006, and M.S. degrees in Electric Electronic Eng. from Korea University of Technology \& Education, in 2008. Since Sept. 2008, he has been a Ph.D. student in Korea University of Technology \& Education. His research interests are wireless sensor networks, cognitive radio.

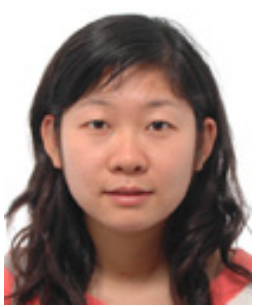

\section{Wenmin Zhu}

She received the B.S. degree in Science and Technology of Electronic Information from Tianjin Polytechnic University, 2008. Since Sept. 2008, she has been a M.S. student in Korea University of Technology \& Education. His research interests are cognitive radio and wireless networks

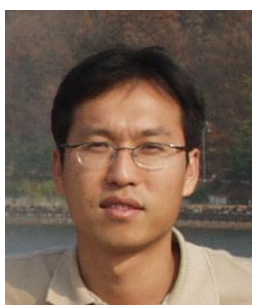

\section{Hyung-Kun Park}

He received the B.S, M.S. and Ph.D. degrees in Electronic Engineering from Korea University, in 1995, 1997, and 2000, respectively. From September 2000 to August 2001, he was a postdotoral fellow at Electrical and Computer Engineering in University of Colorado at Colorado Springs, Colorado, USA. In Sept. 2001, he joined, Hyundai Syscomm, Inc., Ichon, Korea. Since March 2004, he has been with the School of Information Technology at the Korea University of Technology and Education where he is currently an assistant professor. His research interest is radio resource management, packet scheduling, wireless communications. 